\title{
Compact radio sources and jet-driven AGN feedback in the early universe: constraints from integral-field spectroscopy ${ }^{\star}$
}

\author{
N. P. H. Nesvadba ${ }^{1, \dagger}$, M. D. Lehnert ${ }^{1}$, C. De Breuck ${ }^{2}$, A. Gilbert $^{3}$, and W. van Breugel ${ }^{4}$ \\ 1 GEPI, Observatoire de Paris, CNRS, Universite Denis Diderot, 5 Place Jules Janssen, 92190 Meudon, France \\ e-mail: nicole.nesvadba@obspm.fr \\ 2 European Southern Observatory, Karl-Schwarzschild Strasse, 85748 Garching bei München, Germany \\ 3 Institute of Geophysics and Planetary Physics, Lawrence Livermore National Laboratory, 7000 East Avenue, L413, Livermore, \\ CA 94550, USA \\ 4 University of California, Merced, PO Box 2039, Merced, CA 95344, USA
}

Recieved 27 June 2007 / Accepted 21 August 2007

ABSTRACT

\begin{abstract}
Aims. To investigate the impact of radio jets during the formation epoch of their massive host galaxies, we present an analysis of two massive, $\log M_{\text {stellar }} / M_{\odot} \sim 10.6$ and 11.3, compact radio galaxies at $z=3.5$, TNJ0205+2242 and TNJ0121+1320. Their small radio sizes $(R \leq 10 \mathrm{kpc})$ are most likely a sign of youth. In particular, we compare their radio properties and gas dynamics with those in well extended radio galaxies at high redshift, which show strong evidence for powerful, jet-driven outflows of significant gas masses $\left(M \sim 10^{9-10} M_{\odot}\right)$.

Methods. Our analysis combines rest-frame optical integral-field spectroscopy obtained with SINFONI on the VLT with existing radio imaging, $\mathrm{CO}(4-3)$ emission line spectra, and rest-frame UV longslit spectroscopy.

Results. [OIII] $\lambda 5007$ line emission is compact in both galaxies and lies within the region defined by the radio lobes. For TNJ0205+2242, the Ly $\alpha$ profile narrows significantly outside the jet radius, indicating the presence of a quiescent halo. TNJ0121+1320 has two components at a projected relative distance of $\sim 10 \mathrm{kpc}$ and a velocity offset of $\sim 300 \mathrm{~km} \mathrm{~s}^{-1}$, measured from the $[\mathrm{OIII}] \lambda 5007$ velocity map. This suggests that the fainter component is orbiting around the more massive, radio-loud galaxy. If motions are gravitational, this implies a dynamical mass of $2 \times 10^{11} M_{\odot}$ for the radio-loud component.

Conclusions. The dynamical mass, molecular gas mass measured from the $\mathrm{CO}$ line emission, and radio luminosity of these two compact radio galaxies imply that compact radio sources may well develop large-scale, energetic outflows as observed in extended radio galaxies, with the potential of removing significant fractions of the ISM from the host galaxy. The absence of luminous emission line gas extending beyond the radio emission in these sources agrees with the observed timescales and outflow rates in extended radio galaxies, and adds further evidence that the energetic, large-scale outflows observed in extended radio sources (Nesvadba et al. 2006, ApJ, 650, 693) are indeed the result of influence of the radio jet.
\end{abstract}

Key words. galaxies: high-redshift - galaxies: formation - galaxies: kinematics and dynamics - infrared: galaxies - galaxies: jets

\section{Introduction}

Although powerful AGN were the first sources discovered at high redshifts, it is only now becoming clear that they may also have a profound impact on the evolution of their host galaxies, particularly those at the upper end of the galaxy mass function. In order to explain some of the ensemble properties of massive galaxies in the local Universe, such as the slope of the upper end of the mass function (e.g., Benson et al. 2003), galaxy evolution models have incorporated a phase of powerful AGN feedback in the early evolution of massive galaxies (e.g., Silk \& Rees 1998; Hopkins et al. 2005; Croton et al. 2006; Di Matteo et al. 2005). Such a phase effectively terminates star-formation in already formed massive galaxies by heating and removing significant fractions of the cold gas within the host galaxy.

However, direct observational evidence for strong AGN driven (negative) feedback is still very rare. Although AGN undoubtedly maintain enormous energy outputs over their lifetimes of $10^{7-8}$ yrs (Martini 2004), we do not know by which physical

* Based on observations collected at the European Southern Observatory, Very Large Telescope Array, Cerro Paranal, Chile (076.A-0684(A)).

${ }^{\dagger}$ Marie Curie Fellow. mechanism this energy is transferred into kinetic energy of the interstellar medium (ISM) of the host galaxy - and whether any such mechanism exists that could provide the necessary large coupling efficiencies.

Radio jets in high-redshift radio galaxies are known to strongly distort the gas kinematics along the jet axis (e.g., McCarthy et al. 1996; Tadhunter 1991; Villar-Martín et al. 1999), and may therefore be a candidate mechanism to ensure efficient coupling between the AGN and the host galaxy - if the influence of the jet is not confined to the gas along the jet axis. Due to the complexity of the gas dynamics in high-redshift radio galaxies, this is difficult to determine from longslit spectra alone, but requires integral-field spectroscopy. Nesvadba et al. (2006) find from rest-frame optical integral-field spectroscopy of the $z=$ 2.2 powerful radio galaxy MRC1138-262 that this may indeed be the case. They identify massive outflows of $\sim 10^{9} M_{\odot}$ of ionized hydrogen, with relative velocities of up to $>2000 \mathrm{~km} \mathrm{~s}^{-1}$ and emission line FWHMs $>1000 \mathrm{~km} \mathrm{~s}^{-1}$, which do not appear to be strongly collimated along the axis of the radio jet. However, Nesvadba et al. find that dynamical timescale and energy arguments nonetheless favor the radio jet as being the dominant mechanism accelerating the gas, estimating that over a lifetime of the outflow, $t_{\text {dyn }} \sim 10^{7}$ yrs, a total of $E_{\text {kin }} \gtrsim 10^{60} \mathrm{erg}$ 
Table 1. Emission lines in TNJ0205+2242.

\begin{tabular}{|c|c|c|c|c|c|}
\hline $\begin{array}{l}\text { Line } \\
\text { (1) }\end{array}$ & $\begin{array}{l}\lambda_{0} \\
(2)\end{array}$ & $\begin{array}{l}\lambda_{\mathrm{obs}} \\
\text { (3) }\end{array}$ & $\begin{array}{c}z \\
(4)\end{array}$ & $\begin{array}{c}F W H M \\
(5)\end{array}$ & $\begin{array}{c}\text { Flux } \\
(6)\end{array}$ \\
\hline$\overline{\text { [OIII] }}$ & 5007 & $2.25754 \pm 0.00045$ & $3.50867 \pm 0.00070$ & $143 \pm 10$ & $2.7 \pm 0.19$ \\
\hline [OIII] & 5007 & $2.25608 \pm 0.00045$ & $3.50574 \pm 0.00070$ & $1171 \pm 72$ & $23.5 \pm 1.4$ \\
\hline $\mathrm{H} \beta$ & 4861 & $2.19179 \pm 0.00054$ & $3.50882 \pm 0.00086$ & $1699 \pm 145$ & $3.8 \pm 0.37$ \\
\hline
\end{tabular}

Column (1) - Line. Column (2) - Rest-frame wavelength. Column (3) - Observed wavelength. Column (4) - Redshift. Column (5) - FWHM, corrected for instrumental resolution. Column (6) - Line flux in units of $10^{-17} \mathrm{erg} \mathrm{s}^{-1} \mathrm{~cm}^{-2}$.

Table 2. Emission lines in TNJ0121+1320.

\begin{tabular}{lcccccc}
\hline \hline $\begin{array}{l}\text { Component } \\
(1)\end{array}$ & $\begin{array}{c}\text { Line } \\
(2)\end{array}$ & $\begin{array}{c}\lambda_{0} \\
(3)\end{array}$ & $\begin{array}{c}\lambda_{\text {obs }} \\
(4)\end{array}$ & $\begin{array}{c}z \\
(5)\end{array}$ & $\begin{array}{c}F W H M \\
(6)\end{array}$ & $\begin{array}{c}\text { Flux } \\
(7)\end{array}$ \\
\hline $\mathrm{A}$ & {$[\mathrm{OIII}]$} & 5007 & $2.26322 \pm 0.00045$ & $3.52001 \pm 0.0007$ & $724 \pm 44$ & 13.2 \\
$\mathrm{~A}$ & $\mathrm{H} \beta$ & 4861 & $2.19864 \pm 0.00047$ & $3.52293 \pm 0.0008$ & $394 \pm 55$ & 1.0 \\
$\mathrm{~B}$ & {$[\mathrm{OIII}]$} & 5007 & $2.26541 \pm 0.00046$ & $3.52449 \pm 0.0007$ & $274 \pm 21$ & 2.4 \\
$\mathrm{~B}$ & $\mathrm{H} \beta$ & 4861 & $2.20054 \pm 0.00047$ & $3.52692 \pm 0.0008$ & $153 \pm 47$ & 0.3 \\
\hline
\end{tabular}

Column (1) - Component. Column (2) - Line. Column (3) - Rest-frame wavelength. Column (4) - Observed wavelength. Column (5) - Redshift. Column (6) - FWHM, corrected for instrumental resolution. Column (7) - Line flux in units of $10^{-17} \mathrm{erg} \mathrm{s}^{-1} \mathrm{~cm}^{-2}$.

of kinetic energy must have been injected into the ISM to produce the observed kinematics. For powerful radio galaxies like MRC1138-262, this energy is well above those typically estimated for starburst-driven outflows, or even mergers, and may be sufficient to unbind significant gas fractions from the dark matter halo of a massive galaxy. Since $z \sim 2-3$ HzRGs appear to be massive galaxies experiencing rapid growth of their stellar mass, this suggests that jet-driven AGN feedback as observed in HzRGs may be a promising mechanism to explain the particular characteristics of massive galaxies at low redshift.

Compact high-redshift radio galaxies are particularly promising targets to further investigate whether radio jets may cause large-scale outflows. A radio source may be compact either as a result of a particularly dense ISM, so that the radio jet takes a substantial amount of time to break out, or perhaps not even break out of the ISM of the host galaxy ("frustrated jets"; van Breugel 1984), or are of particularly young ages (Blundell \& Rawlings 1999). Radio spectral ages (e.g., Murgia et al. 1999) and dynamical timescales (Owsianik \& Conway 1998) now indicate that the latter appears to be the case; compact radio sources seem to be particularly young, with ages of at most a few $\times 10^{6}$ yrs (see also Blundell \& Rawlings 1999). This makes them particularly interesting for determining whether or not radio jets can drive large-scale outflows, as the timescales of nuclear activity seem to be significantly shorter than the timescales of starbursts and galaxy mergers $\left(\sim 10^{8}\right.$ yrs; e.g., Barnes \& Hernquist 1996).

Compact radio galaxies may help us in several ways in deciding whether radio jets are indeed the agents in generating the large-scale outflows seen in extended powerful radio galaxies. First, are the emission line regions in these galaxies compact or extended? If the line emission in these sources is related to a recent or on-going merger, then we do not expect to find a correlation between the extended line emission and the size and orientation of the radio source. In particular the emission line region should not exclusively lie within the region defined by the radio jets. Second, how do the kinematics of the emission line gas relate to the region of the radio emission? Compact line emission with similar kinematics as found in the extended radio galaxies and lying within the radio emission will be a sign that the radio jets play a major role for the gas kinematics in the compact source. If the radio jet is the agent, then the kinematics of the emission line gas outside of the region of radio emission should be quiescent, only reflecting gravitationally driven motions. This is indeed what is being observed in the rest-frame UV emission of extended radio galaxies at high redshift with optical longslit and integral-field spectroscopy (e.g., Villar-Martín et al. 2003, 2007). The reason is that the hotspot advance speed is well beyond that expected for both a Compton heated bubble or advance shocks, if either exist in these powerful AGN. Comparing these galaxies to extended radio sources is crucial, as the absence of high surface brightness gas, extended over several $10 \mathrm{~s}$ of kpc in the compact sources will significantly strengthen the idea that the jets in the extended radio galaxies entrained and accelerated significant gas masses. Consequently, we should observe smaller amounts of ionized gas confined within the region of the radio emission in compact radio galaxies because the timescales are so short in the compact sources that they have not had time to entrain as much gas as the large scale radio sources. Previous studies of compact radio sources based on longslit data and at somewhat lower redshift, $z \sim 1$, suggested that this is indeed the case (e.g. Jarvis et al. 2001; Inskip et al. 2002a,b; Best et al. 2000).

Compelling evidence for radio jet-driven outflows in galaxies with compact radio sources, sometimes with velocities of up to $V \geq 1000 \mathrm{~km} \mathrm{~s}^{-1}$ and multiple components with different kinematics, was found in longslit and imaging spectroscopic studies at relatively low redshift $(z \leq 0.5$; e.g., Tadhunter et al. 2001; Holt et al. 2003, 2007; Inskip et al. 2007; Tadhunter 2007). Capetti et al. (1999) used the Hubble Space Telescope Faint Object Camera to obtain imaging and longslit spectra of the narrow-line region of the low-redshift radio-loud Seyfert galaxy Mrk 3 at a spatial resolution of $25 \mathrm{pc}$. They find strong evidence for radio jet-driven outflows in the ionized gas. They propose that the gas is being accelerated and entrained as the overpressurized cocoon of hot X-ray emitting gas surrounding and energized by the radio jet expands. However, at low redshift, the accelerated gas masses, at least in ionized gas, do not appear sufficient to have a large impact on the evolution of the host galaxy, as required in cosmological models (Tadhunter 2007), although a considerable fraction of the total gas mass may be in other phases that are more difficult to observe. Morganti et al. (2005) find outflows of neutral hydrogen implying mass outflow 
rates of up to $50 M_{\odot} \mathrm{yr}^{-1}$, while, e.g., Pounds et al. (2003) argue for outflows of hot X-ray emitting gas.

Complementing these studies, and continuing our analysis of AGN-driven outflows in radio galaxies at high redshift, we present an analysis of two compact, steep-spectrum radio galaxies near the redshift where the co-moving space density of powerful AGN was highest, $z \sim 2-4$ (e.g., Willott et al. 2001). Direct high-redshift studies are essential in understanding the interplay between the radio jets and the ISM at the epoch when AGN were most powerful, massive galaxies were generally younger, and where AGN likely had the greatest impact on galaxy evolution. With their extreme star-formation rates of $\geq 1000 M_{\odot} \mathrm{yr}^{-1}$ and molecular gas masses of a few $\times 10^{10} M_{\odot}$ (e.g., Papadopoulos et al. 2000), radio galaxies at $z \geq 1$ seem to have fundamentally richer ISM than their lower-redshift counterparts, which appear to be poorer in gas, particularly in molecular gas (O'Dea et al. 2005).

We obtained integral-field spectroscopy of the rest-frame optical emission line gas of TNJ0121+1320 and TNJ0205+2242 at $z \sim 3.5^{1}$, which were taken from the sample of De Breuck et al. (2000). Both are compact steep-spectrum sources with radii of $2 \mathrm{kpc}$ and $10 \mathrm{kpc}$ at $8.5 \mathrm{GHz}$, respectively. Their radio power at $1.4 \mathrm{GHz}$ is very similar $P_{1.4}=2 \times 10^{25}$ W. $K$-band imaging of TNJ0121+1320 shows two knots separated by $1.5^{\prime \prime}$, whereas TNJ0205+2242 appears compact in the $K$-band, and is marginally extended along the axis of the radio jet (De Breuck et al. 2001). De Breuck et al. (2003) obtained CO(4-3) emission line data on TNJ0121+1320. The molecular line emission does not appear spatially resolved; the spatial resolution suggests a size of $\leq 4 \mathrm{kpc}$, while they find a line width of $F W H M \sim$ $700 \mathrm{~km} \mathrm{~s}^{-1}$. De Breuck et al. (2001); Humphrey et al. (2007) discuss the $\operatorname{Ly} \alpha$ emission from the two sources. Both galaxies have luminous rest-frame UV emission; TNJ0121+1320 appears compact, whereas TNJ0205+2242 appears surrounded by a halo of narrow Ly $\alpha$ emission extending to $\geq 60 \mathrm{kpc}$.

\section{Observations and data reduction}

We observed TNJ0205+2242 and TNJ0121+1320 in the $K$ band with the integral-field spectrograph SINFONI (Bonnet et al. 2004) on the VLT in December 2005 under good conditions. Both targets are at redshifts $z \sim 3.5$, so that our observations cover the wavelengths of [OIII] $\lambda \lambda 4959,5007$ and $\mathrm{H} \beta$. SINFONI is an image-slicing integral-field spectrograph, with $8^{\prime \prime} \times 8^{\prime \prime}$ field of view at a sampling of $0.25^{\prime \prime} \times 0.25^{\prime \prime}$ pixel scale, and spectral resolution of $R \sim 4000$ in the $K$ band. Individual exposure times were $600 \mathrm{~s}$, and we obtained a total of $10800 \mathrm{~s}$ and $7200 \mathrm{~s}$ of on-source exposure time for TNJ0205+2242 and TNJ0121+1320, respectively.

We used the IRAF (Tody 1993) standard tools for the reduction of longslit-spectra, modified to meet the special requirements of integral-field spectroscopy, and complemented by a dedicated set of IDL routines. Data are dark-frame subtracted and flat-fielded. The position of each slitlet is measured from a set of standard SINFONI calibration data, measuring the position of an artificial point source. Rectification along the spectral axis and wavelength calibration are done before night sky subtraction to account for some spectral flexure between the frames. Curvature is measured and removed using exposures of an arc

${ }^{1}$ Using the flat $\Omega_{\Lambda}=0.7$ cosmology with $H_{0}=70 \mathrm{~km} \mathrm{~s}^{-1} \mathrm{Mpc}^{-1}$ leads to a luminosity distance $D_{\mathrm{L}}=30.6 \mathrm{Gpc}$ and angular size distance $D_{\mathrm{A}}=1.5 \mathrm{Gpc}$. The size scale is $7.3 \mathrm{kpc} /{ }^{\prime \prime}$. The age of the universe for this redshift and cosmological model is $1.8 \mathrm{Gyr}$. lamp, before shifting the spectra to an absolute (vacuum) wavelength scale with reference to the $\mathrm{OH}$ lines in the data. To account for variations in the night sky emission, we normalize the sky frame to the average of the object frame separately for each wavelength before sky subtraction, masking bright foreground objects, and correcting for residuals of the background subtraction and uncertainties in the flux calibration by subsequently subtracting the (empty sky) background separately from each wavelength plane.

The three-dimensional data are then reconstructed and spatially aligned using the telescope offsets as recorded in the header within the same sequence of 5-6 dithered exposures (about one hour of exposure), and by cross-correlating the line images from the combined data in each sequence of exposures, to eliminate relative offsets between different sequences. Telluric correction is applied to each individual cube before the cubes are combined from the several sets of sequences taken for each source. The relative flux calibrations are obtained from observations of standard stars. From the light profile of the standard star (taken after each sequence of exposures or one per approximately every hour), we measure the $F W H M$ spatial resolution in the combined cube.

TNJ0205+2242 is sufficiently close to an $R=14.8 \mathrm{mag}$ bright star, allowing for adaptive optics-corrected observations. With strongly varying coherence times, we obtained Strehl ratios $\sim 0.2-0.7$ (as given in the frame headers), resulting in an overall spatial resolution of $F W H M=0.45^{\prime \prime} \times 0.4^{\prime \prime}$ in right ascension and declination in the final cube, respectively. During our adaptive-optics assisted observations with SINFONI, we monitored the point spread function by making intermittant observations of a nearby bright star. We used the combined image of this star to estimate the size of the seeing disk in our data. Our seeing limited data set of TNJ0121+1320 has a FWHM resolution of $0.65^{\prime \prime} \times 0.55^{\prime \prime}$ in right ascension and declination respectively, measured from the PSF of the standard star taken during the observations.

\subsection{Complementary data sets and relative astrometry}

We complement our SINFONI data with the $8.4 \mathrm{GHz}$ radio data of De Breuck et al. (2002) for TNJ0121+1320, and new, deep 8.4 GHz VLA A-array maps of TNJ0205+2242 with a spatial resolution of $\sim 0.4^{\prime \prime}$. We also use rest-frame UV spectroscopy, which was initially described in De Breuck et al. (2001), to compare the rest-frame optical emission line properties with those of the Ly $\alpha$ emission.

Studying the interplay between radio jets and the ISM of high-redshift galaxies requires an alignment that is accurate to $\leq 0.5^{\prime \prime}$, which is beyond the pointing accuracy of the VLT. TNJ0121+1320 is not resolved at $8.4 \mathrm{GHz}$, and consists of 2 compact components in rest-frame optical continuum and emission line images. Since the position of the radio peak, taken at face value, falls close to the center of the brighter peak in the $K$ band image of TNJ0121+1320, we place the radio source into the center of this component. Moreover, the redshift and line width of the [OIII] $\lambda 5007$ line emission of component $A$ are the same as the $\mathrm{CO}(4-3)$ emission line redshift and line width measured by De Breuck et al. (2003), which also suggests that component $A$ dominates the overall mass budget of the system and is more likely to host the AGN.

To align the radio and infrared data of TNJ0205+2242, we use the radio core, which is detected in our deep A-array imaging at $8.4 \mathrm{GHz}$. In the infrared data, we find an unresolved narrow line region, and broad $\mathrm{H} \beta$ emission at the same position. 

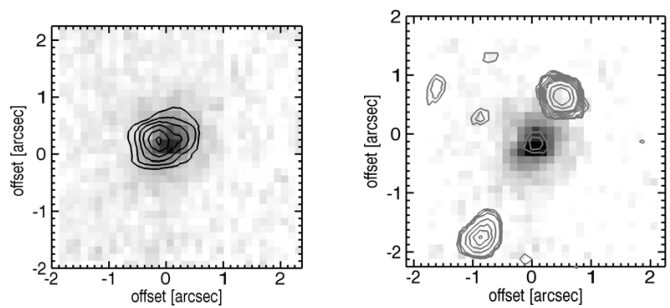

Fig. 1. The left panel shows the [OIII] $\lambda 5007$ emission line morphology of TNJ0205+2242 as grey scale with the continuum morphology overlaid as contours. Contours are given in steps of $2 \sigma$, starting at $3 \sigma$. The $\sim 0.25^{\prime \prime}$ relative offset between the two continuum peaks is significant, because both images were extracted from the same data cube. The right panel shows the same [OIII] $\lambda 5007$ emission line image, but the contours now indicate the $8.4 \mathrm{GHz}$ radio morphology. North is up, east to the left in both images.

Since these are the typical rest-frame optical features of an AGN, we place the radio core at the peak of the narrow-line region. Although this placement is justifiable on astrophysical grounds and in analogy with what is known about radio galaxies generally and other types of powerful AGN, we note that the exact alignment of the radio at the level of a few tenths of an arcsecond has no impact on the overall conclusions presented here.

\section{TNJ0205+2242}

\section{1. [OIII] emission line and continuum morphology}

We show the morphology of the [OIII] $\lambda 5007$ emission line regions of TNJ0205+2242 in the left panel of Fig. 1 with the continuum morphology shown as contours. The line emission is spatially offset from the continuum peak by about $0.25^{\prime \prime}$ to the south-west, which is significant because both images were extracted from the same data cube. Continuum emission is faint, $K=21.6 \pm 0.3$ mag within a box aperture with a length of $1.5^{\prime \prime}$ on either side. We use the FWHM of the continuum image to approximate the radius of the continuum emitting region and assume that the observed full width at half maximum of the continuum is a quadratic sum of the intrinsic $F W H M$ of the source and the $F W H M$ of the seeing disk. We find a seeing corrected full width at half maximum of the source of $0.6^{\prime \prime}$, corresponding to about $4.5 \mathrm{kpc}$.

[OIII] $\lambda 5007$ line emission is only marginally resolved, and elongated along an axis stretching from north-west to south-east, that is, along the axis of the radio jet. Assuming that the intrinsic source $F W H M$ and the seeing $F W H M$ add in quadrature, we estimate the $F W H M$ of the emission line region to be $1.06^{\prime \prime} \times 0.7^{\prime \prime}$ $(8 \mathrm{kpc} \times 5 \mathrm{kpc})$ along the radio jet axis and perpendicular to it, respectively.

\subsection{Integrated spectrum}

The integrated spectrum of TNJ0205+2242 is shown in Fig. 2. The blue lines indicate Gaussian fits to the line profiles. We constrained both [OIII] $\lambda 4959,5007$ lines to have the same redshift and line widths, and imposed a flux ratio $R_{4959,5007}=$ 0.33 . The [OIII] $\lambda 4959,5007$ doublet is well described with two Gaussian components. The narrow component is at a redshift $z=3.50867 \pm 0.0007$, and has a $F W H M=143 \pm 10 \mathrm{~km} \mathrm{~s}^{-1}$. The broad component is blueshifted by $195 \pm 5 \mathrm{~km} \mathrm{~s}^{-1}$ relative to the narrow component, with a width of $F W H M=1171 \pm 71 \mathrm{~km} \mathrm{~s}^{-1}$.

$\mathrm{H} \beta$ line emission is significantly broader than [OIII], although the exact line properties are difficult to determine, due

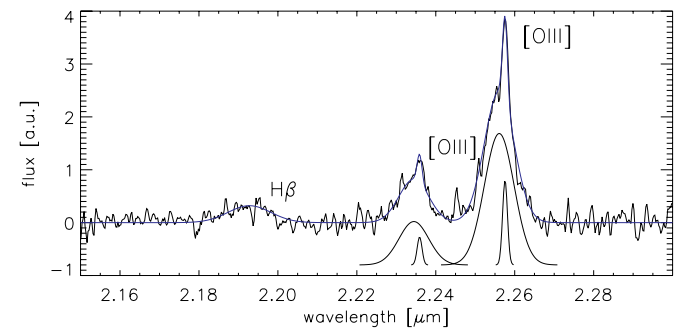

Fig. 2. Integrated $K$-band spectrum of TNJ0205+2242. The blue line indicates the Gaussian fit to the line profile. Black Gaussian curves (shifted by an arbitrary amount along the ordinate) show the individual fit components.

to the faintness of the signal in each spectral bin, and several superimposed $\mathrm{OH}$ lines. The uncertainty is estimated from Monte-Carlo simulations taking into account the signal to noise ratio, line width, and spectral binning, but not the superposition of the $\mathrm{OH}$ line residuals, which are difficult to model precisely. We therefore caution that we may somewhat underestimate the uncertainties of the $\mathrm{H} \beta$ measurement, in particular the line width.

Our best estimate, based only on pixels that are not affected by $\mathrm{OH}$ lines, implies a Gaussian width of $F W H M=1699 \pm$ $145 \mathrm{~km} \mathrm{~s}^{-1}$ and a redshift $z=3.50882 \pm 0.00086$, which is similar to the redshift of the narrow [OIII] $\lambda 5007$ component within the uncertainties. The $F W H M \sim 1700 \mathrm{~km} \mathrm{~s}^{-1}$ may appear large for a high-redshift galaxy, and is near the dividing line to quasars (e.g., Sulentic et al. 2000, find that nuclear broad lines in radio-loud quasars can be as narrow as $F W H M \sim 2000 \mathrm{~km} \mathrm{~s}^{-1}$ ). However, similar $F W H M$ s have also been observed in HzRGs (Iwamuro et al. 2003, find an average $F W H M(\mathrm{H} \beta)=1530 \mathrm{~km} \mathrm{~s}^{-1}$ in their sample of $15 z=$ 2-2.6 HzRGs). This and the faintness of the radio core in TNJ0205+2242 suggest that this galaxy is indeed a radio galaxy rather than a quasar.

The line flux is $3.8 \pm 0.4 \times 10^{-17} \mathrm{erg} \mathrm{s}^{-1} \mathrm{~cm}^{-2}$. Broad $\mathrm{H} \beta$ line emission is detected within an area of approximately one seeing disk surrounding the peak of the [OIII] $\lambda 5007$ emission line region shown in Fig. 1. All line widths are corrected for the instrumental resolution, which we determined from the measured width of night-sky lines from a cube combined in the same way as for the cubes for the radio galaxy.

\subsection{Spatially resolved gas kinematics}

The very regular integrated spectrum does not reflect an overall uniform kinematics of emission line gas, as seen from Fig. 3. To construct these maps, we extracted individual spectra from 5 pixels $\times 5$ pixels $\left(0.6^{\prime \prime} \times 0.6^{\prime \prime}\right)$ box apertures and simultaneously fitted line profiles with up to 4 Gaussian components to the [OIII] $\lambda 5007$ emission line in each spectrum, requiring $S / N>5$ for each component.

We identify three emission line regions with different kinematics and morphologies. (1) A component with a biconal structure, which has a clear $\left(197 \pm 47 \mathrm{~km} \mathrm{~s}^{-1}\right)$ velocity difference between the two sides. It is elongated along the axis of the radio jet and extends to the northern radio lobe. Lines are narrow, with $\sigma \sim 118 \mathrm{~km} \mathrm{~s}^{-1}$. We will in the following use the midpoint between the average velocity in the two sides as reference velocity for the other components. (2) The broadest component is distributed symmetrically around the peak of the line image in Fig. 1 with a spatial extent of approximately one seeing disk. The width of this component is $F W H M=1083 \pm 106 \mathrm{~km} \mathrm{~s}^{-1}$. 

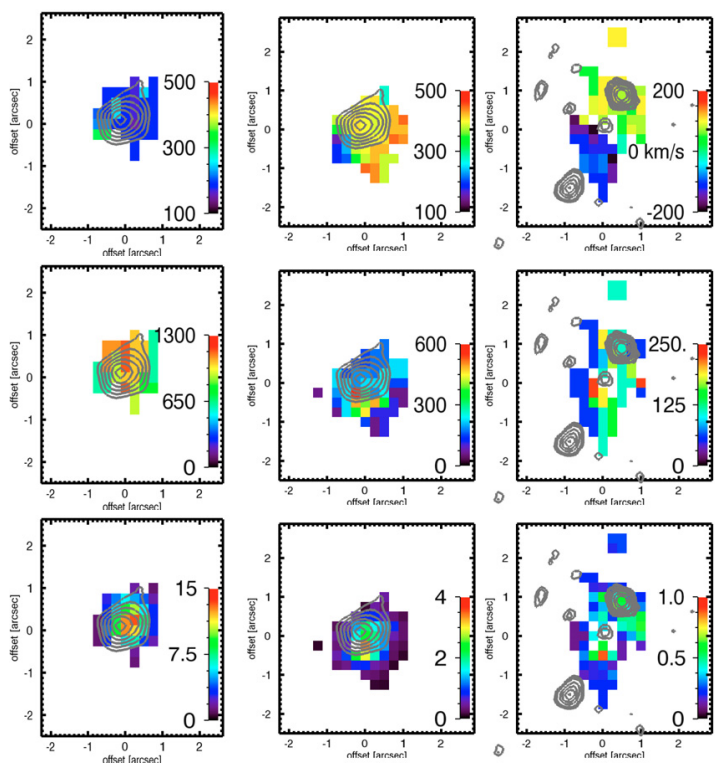

Fig. 3. Maps of relative velocities (top), FHWMs (middle), and line fluxes (bottom) of the 3 line components in TNJ0205+2242, all measured from [OIII] $\lambda 5007$. Contours indicate the $K$ band continuum emission in the left and the mid panel, respectively. Contours in the right panel indicate the $8.4 \mathrm{GHz}$ emission. Colorbars show the velocities and $F W H M \mathrm{~s}$ in $\mathrm{km} \mathrm{s}^{-1}$, and fluxes (in arbitrary units), in the top, middle, and bottom panels, respectively.

The uncertainty in this and the following estimates includes the $1 \sigma$ scatter about the mean. Velocities are uniform in this component, $v=197 \pm 37 \mathrm{~km} \mathrm{~s}^{-1}$. (3) A narrow line component with a similar radius and a range $v=389 \pm 91 \mathrm{~km} \mathrm{~s}^{-1}$. Both regions (2) and (3) are spatially unresolved and likely represent the narrow line region of the AGN.

\subsection{Constraining the mass of TNJ0205+2242}

Due to the complexity of the dynamics of high-redshift radio galaxies, it is in most cases not possible to use their internal kinematics for dynamical mass estimates, as those are not dominated by the large-scale gravitational motion (but see Sect. 4.4 for an exception). However empirically, lines originating from the narrow line regions in AGN are found to correlate with the dynamics of the bulge, e.g. the [OIII] $\lambda 5007$ luminosities (e.g., Kauffmann et al. 2003) or [OIII] $\lambda 5007$ dispersions (e.g. Nelson $\&$ Whittle 1996). Since absolute flux calibrations in the near infrared are inherently uncertain, and we are not able to account for extinction, we will in the following use the [OIII] $\lambda 5007$ emission line dispersion measured from the narrow component of the integrated spectrum of TNJ0205+2242, $\sigma_{\text {[OIII] }}=62 \mathrm{~km} \mathrm{~s}^{-1}$, and the relationship of Nelson \& Whittle (1996). For the measured [OIII] dispersion the Nelson \& Whittle correlation suggests a stellar velocity dispersion of the host, $\sigma_{\text {host }}^{0205} \sim 85 \mathrm{~km} \mathrm{~s}^{-1}$, with a scatter between $\sim 55-136 \mathrm{~km} \mathrm{~s}^{-1}$. Two narrow components contribute to the narrow line in the integrated spectrum of TNJ0205+2242 (Sect. 3.3), but the total narrow line flux is dominated by the spatially unresolved narrow-line region (see Fig. 3). Using the best-fit estimate of Nelson \& Whittle (1996) to approximate the velocity dispersion of TNJ0205+2242, we give a rough mass estimate by setting,

$M_{\mathrm{host}}^{0205}=\frac{5 \sigma^{2} R}{G}=1.2 \times 10^{10}\left(\frac{V}{100 \mathrm{~km} \mathrm{~s}^{-1}}\right)^{2} \frac{R}{\mathrm{kpc}}\left[M_{\odot}\right]$ with the velocity dispersion $\sigma$, and radius $R$ of the host (which we approximate by the half-light radius of the emission line region of TNJ0205+2242, $R \sim 3 \mathrm{kpc}$ ), and the gravitational constant, $G$. For TNJ0205+2242, adopting the best-fit $\sigma$ in the Nelson \& Whittle correlation as given, we estimate $M_{\text {host }}^{0205} \sim 3 \times$ $10^{10} M_{\odot}$. However, we caution that the astrophysical uncertainties of this estimate are large including, e.g., the size estimate, scatter in the Nelson \& Whittle correlation, and possible evolutionary effects. Nonetheless, this suggests that a mass estimate in the range of few $\times 10^{10} M_{\odot}$ is a reasonable estimate.

Photometric masses of HzRGs are also difficult to estimate, as the AGN contaminates the continuum emission by a degree that is hard to constrain, especially with ground-based observations. Seymour et al. (2007) obtained stellar mass estimates for a sample of $69 \mathrm{HzRGs}$ with compact and extended radio sources, for which they obtained Spitzer IRAC and MIPS data, allowing them to constrain the AGN contribution to the SEDs, and in particular through measuring the $1.6 \mu \mathrm{m}$ peak of the stellar contribution, which is suitable to the stellar mass of passively evolving systems with ages $>1$ Gyr. While this may overestimate the age of HzRGs, it does provide an upper limit to the stellar mass in these systems, since the mass-to-luminosity ratio will increase as the stellar population grows older. For TNJ0205+2242 specifically, they estimate $M_{\text {stellar }}^{0205}<6 \times 10^{10} M_{\odot}$, which, given the large uncertainties of either estimate is in good agreement with our results.

\section{TNJ0121+1320}

\section{1. [OIII] emission line and continuum morphology}

The morphology of the [OIII] $\lambda 5007$ emission line region of the second source, TNJ0121+1320, is shown in Fig. 4. The line-free continuum is shown as contours. TNJ0121+1320 consists of two components in the line image. We will in the following refer to the brighter eastern emission line region as component " $A$ ", and to the fainter western region as component " $B$ ".

The projected distance between the two components is $\sim 1.4^{\prime \prime}$, corresponding to $\sim 10 \mathrm{kpc}$. This is well above the seeing disk, which has a $F W H M=0.65^{\prime \prime} \times 0.55^{\prime \prime}$ in right ascension and declination, respectively. Contours in the left panel show the line-free $K$ band continuum of TNJ0121+1320. The total $K$ band magnitude extracted from a $3^{\prime \prime} \times 2^{\prime \prime}$ aperture in RA and Dec respectively, covering components $A$ and $B$ is $K=19.1 \pm 0.3 \mathrm{mag}$, consistent with the $K=18.8$ mag measured by De Breuck et al. (2002). After correcting for contamination with the luminous [OIII] $\lambda 4959,5007$ emission lines from component $A$, we find $K=19.8 \pm 0.4 \mathrm{mag}$. Note that the spatial extent of the continuum and line emission are very similar.

Estimating the contribution of the continuum in component $B$ is more difficult, because our spectra are not deep enough to detect this component in the continuum. Comparing the measured flux of $\mathrm{H} \beta$ and $[\mathrm{OIII}] \lambda 4959,5007$ from component $B$ in our spectrum, $F_{\text {[OIII],H } \beta}=3.7 \times 10^{-17} \mathrm{erg} \mathrm{s}^{-1} \mathrm{~cm}^{-2}$, with the $K$-band flux density of component $B$ measured in a $0.6^{\prime \prime}$ aperture from the $K$-band image of De Breuck et al. (2004), $F_{K, \mathrm{BB}}=4.7 \times 10^{-20} \mathrm{erg} \mathrm{s}^{-1} \mathrm{~cm}^{-2} \AA^{-1}$ (or $22.3 \mathrm{mag}$ ) we find that the line emission contributes at most $20 \%$ to the total $K$-band flux measured from the image. Note that we scaled the $K$-band broad band data to the magnitude measured with SINFONI to ensure that both datasets have the same calibration. Without this correction, the emission line flux would contribute $<15 \%$ to the $K$-band magnitude of component $B$. 

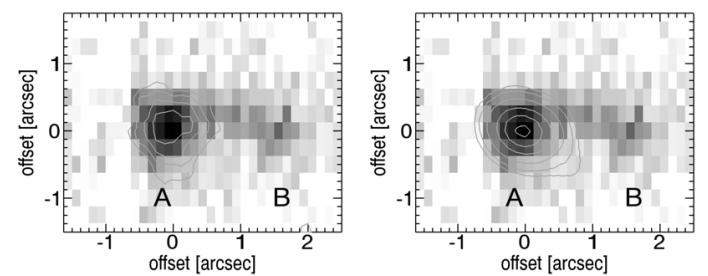

Fig. 4. The left panel shows the [OIII] $\lambda 5007$ emission line morphology of TNJ0121+1320 as grey scale with the continuum morphology overlaid as contours. Contours are shown in steps of $2 \sigma$, starting from $3 \sigma$. The right panel shows the same [OIII] $\lambda 5007$ emission line image, but the contours now indicate the $8.4 \mathrm{GHz}$ radio morphology. North is up, east to the left in both images.

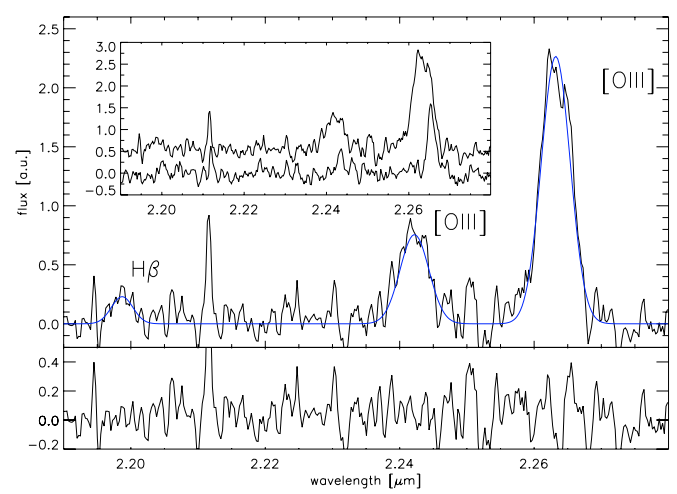

Fig. 5. Upper panel: integrated $K$-band spectrum of component A of TNJ0121+1320. The blue line indicates the Gaussian fit to the line profile, fit residuals are shown in the lower panel. The inset shows the integrated spectrum of component $B$ and the Gaussian fit. The spectrum of component $A$ is shown for comparison, and shifted along the ordinate by an arbitrary amount. All spectra are extracted from 1"square apertures.

Contours in the right panel of Fig. 4 indicate the $8.4 \mathrm{GHz}$ non-thermal radio emission of TNJ0121+1320. The radio source is unresolved and is centered on component $A$ (Sect. 2.1).

\subsection{Integrated spectra of components $A$ and $B$, and gas kinematics}

We extracted integrated spectra of each component from 1.0" $\times$ $1.0^{\prime \prime}$ apertures. The large panel of Fig. 5 shows the spectrum of the major component $A$. [OIII] $\lambda 4959,5007$ line emission is detected and is fitted with a Gaussian at a redshift $z=3.52001 \pm$ 0.0007 and a line width $F W H M=724 \pm 44 \mathrm{~km} \mathrm{~s}^{-1}$, corrected for instrumental resolution. We also detect $\mathrm{H} \beta$ at a redshift $z=3.52293 \pm 0.0008$ and a width of $F W H M=394 \pm 55 \mathrm{~km} \mathrm{~s}^{-1}$. [OIII] $\lambda 5007$ line emission in component $B$ has a velocity offset relative to component $A$ of $297 \pm 7 \mathrm{~km} \mathrm{~s}^{-1}$, and a width of $F W H M=274 \pm 21$.

We used [OIII $] \lambda 5007$ extracted from $0.375^{\prime \prime} \times 0.375^{\prime \prime}$ apertures ( 3 pixels $\times 3$ pixels) to trace the velocities and dispersion of the emission line gas. The maps are shown in Fig. 6. As seen in the integrated spectrum, components $A$ and $B$ have relative velocites of $\sim 200 \mathrm{~km} \mathrm{~s}^{-1}$, dispersions in component $B$ are significantly smaller than in component $A$. However, each individual component is not well resolved spatially. Faint, narrow line emission is marginally detected towards the North-West of component $A$.
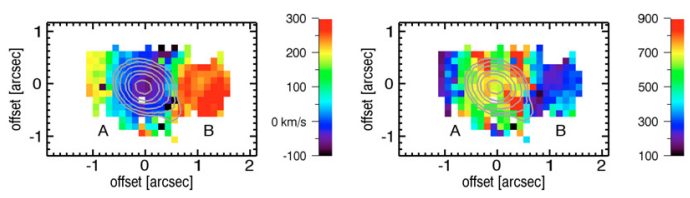

Fig. 6. Velocity map (left) and map of the FHWMs (right) of TNJ0121+1320. The contours indicate the $8.4 \mathrm{GHz}$ radio morphology. Colorbars show relative velocities in $\mathrm{km} \mathrm{s}^{-1}$ and $F W H M \mathrm{~s}$ in $\mathrm{km} \mathrm{s}^{-1}$, respectively. North is up, east to the left in both images.

\subsection{Morphology and kinematics of the ionized and molecular gas}

De Breuck et al. (2003) observed $\mathrm{CO}(4-3)$ line emission in TNJ0121+1320, and measured the redshift and FWHM of the molecular emission, $z_{\mathrm{CO}}=3.520$, and $F W H M_{\mathrm{CO}}=700 \mathrm{~km} \mathrm{~s}^{-1}$, respectively. Within the uncertainties of our measurements, and in particular the low signal-to-noise ratio of the $\mathrm{CO}$ data, the [OIII] $\lambda 5007$ redshift and line width are identical, $z_{[\mathrm{OIII}]}=$ $3.52001 \pm 0.0007$, and $F W H M_{[\mathrm{OIII}]}=724 \pm 44$. Similarly, both $\mathrm{CO}(4-3)$ and [OIII] $\lambda 5007$ line emission appear to also originate from similarly compact regions of the galaxy: the $\mathrm{CO}$ emission line region is not spatially resolved, so we use the $F W H M$ of the beam as an upper limit to the size, $F W H M_{\mathrm{CO}} \leq 0.54^{\prime \prime}$, which corresponds to $\leq 4 \mathrm{kpc}$. Since the emission line region of TNJ0121+1320 A is not spatially resolved, the size of the [OIII] $\lambda 5007$ is dominated by the size of the seeing disk. This implies an upper limit to the size of the line emitting region of of $0.5^{\prime \prime}$, or $\sim 4 \mathrm{kpc}$.

\subsection{Dynamical and molecular mass}

We will now use the smaller component $B$ of TNJ0121+1320 to estimate a dynamical mass for a high-redshift radio galaxy. This component has all the properties expected from a companion galaxy: the $K$-band emission from this source is dominated by the continuum (Sect. 4.1), and the emission lines are spectrally resolved with a velocity, $\sigma_{B}^{0121}=72 \pm 11 \mathrm{~km} \mathrm{~s}^{-1}$. The large difference in velocity dispersion and the velocity offset between component $A$ and $B$ of $297 \pm 7 \mathrm{~km} \mathrm{~s}^{-1}$ make it unlikely that they are part of the same galaxy, although the kinematics suggest that they are physically related, and gravitationally bound. (We note that TNJ0121+1320 B appears overall very similar to the smaller component $J 2$ of the $z=2.6$ submillimeter galaxy SMMJ14011+0252, Nesvadba et al. 2007b). If the dispersion is dominated by the large-scale gravitational potential of TNJ0121+1320 B, then we can use the dispersion to estimate a dynamical mass of component $B$ from Eq. (1), $M_{B}^{0121} \sim 7 \times 10^{9} M_{\odot}$.

The relative velocity between components $A$ and $B$ allows a rough dynamical mass estimate for component $A$. The position of the radio source, the peak of the $\mathrm{CO}$ line emission, and the brighter continuum emission all favor TNJ0121+1320 A to be the more massive component.

We therefore assume that $B$ is orbiting $A$ with a circular velocity $v_{\mathrm{c}, A, B}=297 \pm 10 \mathrm{~km} \mathrm{~s}^{-1}$ similar to the observed velocity offset $v_{\mathrm{obs}}$, and estimate the mass of component $A, M_{A}^{0121}$, setting

$M_{A}^{0121}=\frac{v_{\mathrm{obs}}^{2} D_{A, B}}{G}=2.3 \times 10^{9}\left(\frac{V}{100 \mathrm{~km} \mathrm{~s}^{-1}}\right)^{2} \frac{R}{1 \mathrm{kpc}} M_{\odot}$

where $D_{A, B}=10 \mathrm{kpc}$ is the projected distance between the two components. Not correcting for inclination, we estimate a dynamical mass $M_{A}^{0121}=2 \times 10^{11} M_{\odot}$. Strictly speaking, this is 
only a lower limit; due to the unknown inclination and separation, the intrinsic mass might be factors of a few higher. For example, for the average of a statistical sample, we expect the intrinsic masses to be larger by factors $\sim 2-3$.

TNJ0121+1320 is also part of the sample of Seymour et al. (2007) with photometric mass estimates (see Sect. 3.4 for a discussion of their stellar mass estimate for TNJ0205+2242); they find $M_{\text {stellar }}^{0121} \sim 1.0 \times 10^{11} M_{\odot}$ (in total for components $A$ and $B)$, which is close to our dynamical mass estimate, although the good agreement may be to some degree fortuitous, given the large uncertainties in either estimate. Given that component $B$ is much fainter and appears to have a significantly smaller dynamical mass than component $A$, we assume that the stellar mass budget is dominated by component $A$. Comparing with the average stellar mass of the Seymour et al. sample, $M_{\text {stellar }} \sim 3 \times$ $10^{11} M_{\odot}$, TNJ0121+1320 appears to have a typical mass for HzRGs, unlike TNJ0205+2242, which appears to be somewhat less massive.

The $\mathrm{CO}(4-3)$ measurement of De Breuck et al. (2003), $S_{43} v=1.2 \mathrm{Jy} \mathrm{km} \mathrm{s}^{-1}$, allows us to estimate a molecular gas mass. Following Downes \& Solomon (1998), we adopt $M_{\mathrm{CO}}=$ $0.8 \mathcal{L}_{\mathrm{CO}}^{\prime}$, where $\mathcal{L}^{\prime}$ is the $\mathrm{CO}$ luminosity in units of $\mathrm{K} \mathrm{km} \mathrm{s}^{-1} \mathrm{pc}^{2}$, and find a molecular gas mass of $M_{\text {mol }}^{0121} \sim 4 \times 10^{10} M_{\odot}$. We note that this mass estimate is similar to $\mathrm{CO}$ mass estimates of galaxies with extended radio sources (e.g. Papadopoulos et al. 2000). TNJ0121+1320 is gas rich, which a molecular mass fraction of $\sim 20 \%$.

\section{A unified view of AGN feedback in extended and compact high-z radio galaxies}

\subsection{Constraints from optical emission line regions}

Integral-field spectroscopy of the rest-frame optical emission line gas of $z \geq 2$ radio galaxies with extended radio sources $(D \geq$ $20 \mathrm{kpc}$ ) shows strong evidence for enormous outflows of ionized gas (Nesvadba et al. 2006, 2007a). These outflows have total kinetic energies of $\sim 10^{60}$ erg that appear sufficient to unbind a significant fraction of the ISM of a massive, gas-rich galaxy within the $\sim 10^{7}$ yrs lifetime of a radio jet. Timescale, energy, and geometrical arguments indicate that the outflows are most likely driven by the mechanical energy input of the radio jet, through a mechanism by which the jet couples efficiently to the ISM of the host galaxy. A good candidate mechanism is inflation of an overpressurized cocoon of hot gas energized by and forming around the radio jet, that entrains and accelerates the ambient gas as it expands (Begelman \& Cioffi 1989; Pedlar et al. 1985). Capetti et al. (1999) argue that the morphology and kinematics in the narrow-line region in the low-redshift Seyfert galaxy MRK 3 on scales of a few $100 \mathrm{pc}$ are most likely powered by a inflating cocoon. Such a mechanism would explain the high coupling efficiency that appears necessary to explain radio emission on small scales (see also. e.g., Axon et al. 2000; O'Dea et al. 2002) and on larger scales (Nesvadba et al. 2006, 2007a), as well as the ionization structure of radio galaxies at $z \sim 1$ (Inskip et al. 2002a; Best et al. 2000).

This basic, and certainly over-simplified, scenario finds support from the galaxies with compact radio sources we discuss here. Most obviously, and similar to previous longslit studies of compact HzRGs (Jarvis et al. 2001; Inskip et al. 2002a,b), we do not find bright, extended emission line regions in the compact radio galaxies, that would extend beyond the size of the radio lobes. For any mechanism where the gas is being accelerated by the radio jets, this is certainly reassuring, since the

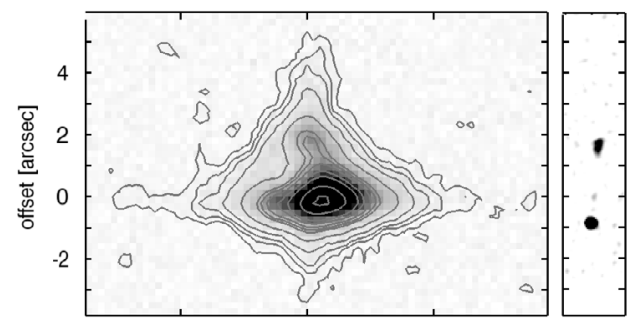

Fig. 7. Left: spatially-resolved, two-dimensional longslit spectrum of the Ly $\alpha$ emission line of TNJ0205+2242. The dispersion axis is along the abscissa. Right: $8.4 \mathrm{GHz}$ VLA map of TNJ0205+2242. Note that the size of the broad Ly $\alpha$ emitting region corresponds approximately to the size of the radio jets, while the gas beyond the radio jets becomes more quiescent, with $F H W M=531 \mathrm{~km} \mathrm{~s}^{-1}$ and a nearly Lorentzian profile.

absence of large-scale outflows is what is expected if the radio jet is the dominant driver of the giant outflows seen in the extended sources, but we may see the compact sources before the jets have driven out sufficiently large gas masses. In the past years, evidence has accumulated that compact radio sources are young relative to extended sources (Owsianik \& Conway 1998; Murgia et al. 1999; Best et al. 2000, based on their spectral properties and direct observations of motion within the jets), but this basic argument does not explicitly rely on this.

\subsection{Evidence for quiescent $L y \alpha$ halos beyond the radio lobes}

In Fig. 7 we compare the radio map of TNJ0205+2242 with the two-dimensional rest-frame $\operatorname{Ly} \alpha$ spectrum. As discussed by De Breuck et al. (2001), Ly $\alpha$ emission extends over $\sim 8^{\prime \prime}$. The size of the inner, perturbed, region (with $F W H M=1550 \mathrm{~km} \mathrm{~s}^{-1}$ compared to $F W H M=531 \mathrm{~km} \mathrm{~s}^{-1}$ in the outer region) corresponds to the size of the radio jets, which in turn mark the spatial extent of the bright [OIII] $\lambda 5007$ emission line regions in Fig. 3. This good correspondence between kinematics, and emission line surface brightness with the extent of the radio jet provides robust evidence that the gaseous outflows of high-redshift radio galaxies are indeed driven by the radio jet. Note that [OIII] does not suffer from scattering and the optical depth effects, that make Ly $\alpha$ a difficult tracer of the kinematics.

The Ly $\alpha$ spectrum of TNJ0205+2242 suggests that compact, steep-spectrum radio galaxies are surrounded by very similar halos as extended radio sources. Villar-Martín et al. (2002, 2003 , 2007) identify $\leq 100-\mathrm{kpc}$ sized, kinematically quiescent Ly $\alpha$ halos surronding $z \sim 2-3$ radio galaxies with extended radio sources, which have $F W H M \sim 500 \mathrm{~km} \mathrm{~s}^{-1}$ and are outside the radius of influence of the radio jets.

Finding a quiescent Ly $\alpha$ halo around a compact HzRG may indicate that these galaxies reside within similarly gas rich, dense environments as extended HzRGs, as traced by the kinematics and size of their turbulent and quiescent Ly $\alpha$ halos, adding an environmental argument to the interpretation that they are their younger analogs.

\section{AGN feedback energy in compact radio galaxies}

Having direct measurements of the dynamical and molecular gas mass (Sect. 4.4) and jet power of TNJ0121+1320 (Sect. 1), we can investigate explicitly, whether the radio jet has the potential to unbind a significant fraction of the ISM from the dark matter halo of the host galaxy.

As in Nesvadba et al. (2006), we use the scalings of Bullock et al. (2001) to estimate that the gas will have to be accelerated 
to an escape velocity of $\sim 700 \mathrm{~km} \mathrm{~s}^{-1}$, if it is to be unbound. This corresponds to a total kinetic energy injection of $E \sim 9.9 \times$ $10^{58} M_{\odot, 10} v_{\text {esc,1000 }}$ erg, that must be supplied by the radio jet. $M_{\odot, 10}$ is the total mass of the outflow in units of $10^{10} M_{\odot}$, the escape velocity, $v_{\mathrm{esc}, 1000}$, is in units of $1000 \mathrm{~km} \mathrm{~s}^{-1}$.

For $M_{\mathrm{mol}}^{0121} \sim 4 \times 10^{10} M_{\odot}$, this corresponds to a total energy injection of $\sim 4 \times 10^{59} \mathrm{erg}$. Can this energy be provided by the radio jet? Similar to Nesvadba et al. (2006), we calculate the jet kinetic energy from the jet luminosity in the frequency range of $0.1-1 \mathrm{GHz}$ in the rest-frame, using the radio fluxes measured by De Breuck et al. (2001), and find $\mathcal{L}_{\text {jet }}=2.9 \times 10^{44} \mathrm{erg} \mathrm{s}^{-1}$. Following De Young (1993); Bicknell et al. (1997), we estimate that the jet luminosity corresponds to about $0.1-10 \%$ of the jet kinetic energy. We adopt the second value for a conservative estimate of the jet input energy, $\dot{E}_{\text {kin,jet }} \sim 3 \times 10^{45} \mathrm{erg} \mathrm{s}^{-1}$, or $E_{\text {jet,total }} \sim 9 \times 10^{59}$ erg in a $\sim 10^{7}$ yrs total jet lifetime. Thus, at face value, the jet may remove the entire molecular gas reservoir from the dark matter halo, if the jet kinetic energy is translated into kinetic energy of the gas with $\geq 40 \%$ efficiency. We estimated a lower bound to the jet kinetic energy, and therefore may have overestimated the actual coupling efficiency by factors of a few. In the extended sources, we find efficiencies of on-order $O(10 \%)$, with considerable scatter.

Unfortunately, we do not have a direct measurement of the molecular gas mass for TNJ0205+2242. However, the overall similarity of the two sources, in particular the similar radio luminosity $\left(2.6 \times 10^{44} \mathrm{erg} \mathrm{s}^{-1}\right)$, suggests that any radio-jet related feedback process will be overall very similar. Moreover, TNJ0205+2242 appears to have a smaller mass than TNJ0121+1320 by factors of a few, so that it may have a somewhat more shallow gravitational potential and a smaller gas mass (Sects. 3.4 and 4.4).

\section{What drives the gas dynamics in compact HzRGs?}

If the radio jets in compact HzRGs are powerful enough to induce similar outflows to those in extended radio sources, does this imply that their gas dynamics are also dominated by the radio jet? Basic causality arguments suggest that this is rather unlikely. Dynamical timescales of the radio jets in compact sources, $\leq 10^{5-6}$ yrs, are less than the sound crossing time of the host $\left(O\left(10^{8} \mathrm{yrs}\right)\right.$ for a sound speed of $\left.O\left(10 \mathrm{~km} \mathrm{~s}^{-1}\right)\right)$.

Comparing with the mass outflow rates in extended HzRGs, we can give an order-of-magnitude estimate of the entrainment rate, and find a similar answer. Since the entrainment rate will depend crucially on the gas content in the host galaxy, which seems to evolve strongly with redshift (Sect. 1), we cannot rely on entrainment rates estimated from detailed jet modelling in compact radio sources at low redshift (e.g. Laing \& Bridle 2002). Assuming an overall constant entrainment rate within a factor $\sim 10$ during the jet lifetime, and using the observed $\mathrm{d} M / \mathrm{d} t \sim$ few $\times 100 M_{\odot} \mathrm{yr}^{-1}$, corresponding to the estimates of Nesvadba et al. (2006, 2007a), a simple scaling can approximate the total mass of entrained material,

$M_{\mathrm{entr}}=f_{\mathrm{c}} f_{\rho_{r}} \frac{\dot{M}_{\mathrm{obs}}}{M_{\odot} \mathrm{yr}^{-1}} \frac{\tau}{10^{6} \mathrm{yrs}}\left[10^{8} M_{\odot}\right]$

with the outflow rate $\dot{M}_{\text {obs }}$ observed in extended radio galaxies (Nesvadba et al. 2006, 2007a), and age of the radio source, $\tau . f_{\mathrm{c}}$ and $f_{\rho(r)}$ are correction factors accounting for the radial density profile of the host galaxy and variations in the jet advance speed, respectively. Readhead et al. (1996) argue that the jet advance speed is probably not a strong function of radius, so that $f_{\mathrm{c}} \sim 1$. For approximately uniform density, $\rho(r) \sim \rho_{0}$, we find that after $10^{6}$ yrs, only few $\times 10^{8} M_{\odot}$ will have been entrained by the radio jet, less than a percent of the $4 \times 10^{10} M_{\odot}$ in molecular mass observed in TNJ0121+1320. The mass outflow rate in extended HzRGs was estimated from the mass of ionized gas only, and certainly underestimates the intrinsic mass outflow rate by factors of a few. Similarly, a non-uniform density profile will imply a larger entrainment rate in young radio sources. Therefore, we likely underestimate the true entrainment rate by factors of a few. However, even for a $\sim 10 \times$ larger entrained gas mass, no more than $\sim 10 \%$ of the molecular gas mass could have been entrained in a compact HzRG. This is a strong contrast to extended HzRGs, which have entrained gas masses similar if not greater than their molecular gas content (Nesvadba et al. 2007a), and may be a simple consequence of the younger ages of compact sources.

There is an interesting trend if we compare the two sources, although statements based on only two galaxies can hardly be conclusive. Using the size of the radio jet to estimate the age of each source, we find that TNJ0205+2242 may be factors of a few larger than the more compact TNJ0121+1320 $\left(t_{\text {jet }}^{0205} \leq 4 \times\right.$ $10^{6} \mathrm{yrs}$ compared to $t_{\text {jet }}^{0121} \leq 7 \times 10^{5} \mathrm{yrs}$, for TNJ0121+1320 and TNJ0205+2242, respectivley, and for a jet advance speed of $v \geq 0.01 c$, following Wellman et al. 1997). A similar trend is seen in the emission line properties of the two sources: TNJ0205+2242 has a biconal component that appears related to the radio jet, as suggested by size and spatial alignment, although it has a smaller velocity gradient, $\sim 200 \mathrm{~km} \mathrm{~s}^{-1}$, than extended radio galaxies ( $\Delta v \geq 1000 \mathrm{~km} \mathrm{~s}^{-1}$ Nesvadba et al. 2007a), and contains only a few percent of the total ionized gas, if the $[\mathrm{OIII}] \lambda 5007 / \mathrm{H} \beta$ line ratios are roughly constant. This galaxy also has an unresolved, broad [OIII] $\lambda 5007$ emission line, which is blueshifted by $\sim 200 \mathrm{~km} \mathrm{~s}^{-1}$ relative to the narrow [OIII] component, and has a line width $F W H M \sim 1200 \mathrm{~km} \mathrm{~s}^{-1}$ similar to extended radio sources. This may indicate a similarly turbulent, but less evolved, outflow as seen in the extended HzRGs. TNJ0121+1320 has more narrow lines $\left(F W H M \sim 700 \mathrm{~km} \mathrm{~s}^{-1}\right.$, which is not unplausibly high for a galaxy with a mass of a few $\times 10^{11} M_{\odot}$ ), and no biconal component. Note that the turbulent line broadening will be mostly isotropic, hence we do not expect differences in viewing angle to have a large impact.

Overall, the properties of each of these two sources are in broad agreement with what would be expected if the gas dynamics of the host galaxies were more and more dominated by the energy injection of the radio jet as the radio source ages and expands: TNJ0205+2242, which has a more extended, and presumably more evolved radio source, resembles galaxies with well extended radio sources more than TNJ0121+1320, whose radio source is very compact, and presumably younger.

\section{Summary}

We presented a study of the rest-frame optical emission line gas of two powerful radio galaxies at $z=3.5$, TNJ0205+2242 and TNJ0121+1320, using integral-field spectroscopy, combined with radio, rest-frame $\mathrm{UV}$, and $\mathrm{CO}$ emission line data. Both galaxies have compact radio emission, with radii $\leq 10 \mathrm{kpc}$. They have bright [OIII] $\lambda 4959,5007$ and $\mathrm{H} \beta$ line emission, and faint, compact continuum emission. The excessive emission line equivalent widths imply that line contamination is a serious complication for studying the continuum morphologies of high-redshift radio galaxies at redshifts where strong 
line emission falls within the bandpass of broad band filters, and adds additional uncertainties in obtaining SEDs of highredshift radio galaxies. Both galaxies have a broad [OIII] $\lambda 5007$, 4959 emission line component, with FWHMs $\sim 700 \mathrm{~km} \mathrm{~s}^{-1}$ and $\sim 1200 \mathrm{~km} \mathrm{~s}^{-1}$ for TNJ0121+1320 and TNJ0205+2242 respectively. TNJ0205+2242 has a narrow component superimposed, which is redshifted relative to the broad component by $\sim 200 \mathrm{~km} \mathrm{~s}^{-1}$.

A secondary component of discrete emission in TNJ0121+1320 offers a unique possibility to estimate the dynamical mass of a high-redshift radio galaxy in the same way as often applied to other galaxy populations. TNJ0121+1320 B, at a distance of $\sim 10 \mathrm{kpc}$ from the massive radio galaxy, and at a velocity offset of $\sim 300 \mathrm{~km} \mathrm{~s}^{-1}$, has all the properties of being a $M \sim 10^{9-10} M_{\odot}$ galaxy that is physically connected to the more massive component, and may be in the process of merging. From the velocity offset and projected distance, we estimate that the major component of TNJ0121+1320 has a dynamical mass of $\sim 2 \times 10^{11} M_{\odot}$ within a radius of $\sim 10 \mathrm{kpc}$. This agrees well with photometric mass estimates of TNJ0121+1320 (Seymour et al. 2007), and suggests that TNJ0121+1320 has a typical mass for high-redshift radio galaxies. For TNJ0205+2242, we estimate a somewhat lower mass of a few $\times 10^{10} M_{\odot}$ from the dispersion of the narrow [OIII] $\lambda 5007$ component, using the empirical relationship between $F W H M$ of the narrow-line region and the stellar velocity dispersion of Nelson \& Whittle (1996). This again agrees well with the photometric mass estimate of Seymour et al. (2007), who find $4 \times 10^{10} M_{\odot}$.

Emission line morphologies in both sources are compact, and do not extend beyond the most extended radio emission. This is a necessary condition for arguing that radio jets may provide a sufficiently efficient coupling mechanism between the AGN and the ISM with the potential of removing significant gas masses from the host galaxy. In particular, this is fully compatible with the conclusion that the large-scale energetic outflows observed in extended radio galaxies appear to be powered predominantly by the radio jet (Nesvadba et al. 2006, 2007a). Similarly, compact radio galaxies have jets that appear powerful enough to unbind significant gas fractions from a massive, gas-rich galaxy, and specifically, to unbind the $4 \times 10^{10} M_{\odot}$ in molecular mass estimated from $\mathrm{CO}(4-3)$ measurements in TNJ0121+1320. Adopting the "paradigm of youth", that the compactness of a radio source is a sign of young age, and estimating entrainment rates from the large-scale outflows we observe in galaxies with extended radio sources, we find that compact radio sources have not yet had the time to entrain significant gas masses. Timescale and causality arguments, as well as the individual properties of the likely more evolved TNJ0205+2242 relative to the likely somewhat younger TNJ0121+1320, are broadly consistent with this picture.

Acknowledgements. The authors wish to thank the anonymous referee for good advice on how to improve the paper. We are grateful to the ESO OPC for their allocation of observing time and the staff at Paranal for their help and support in making these observations. N.P.H.N. wishes to acknowledge financial support from the European Commission through a Marie Curie Postdoctoral Fellowship and M.D.L. wishes to thank the Centre National de la Recherche Scientifique for its continuing support of his research. Part of this work was performed under the auspices of the US department of Energy, National Nuclear Security Administration by the University of California, Lawrence Livermore National Laboratory under contract No. W-7405-Eng-48. W.v.B. acknowledges support for radio and infrared galaxy studies with the Spitzer Space Telescope at UC Merced, including the work reported here, via NASA grants SST GO-1264353, GO-1265551, GO-1279182 and GO-1281587.

\section{References}

Axon, D. J., Capetti, A., Fanti, R., et al. 2000, AJ, 120, 2284

Barnes, J. E., \& Hernquist, L. 1996, ApJ, 471, 115

Begelman, M. C., \& Cioffi, D. F. 1989, ApJ, 345, L21

Benson, A. J., Bower, R. G., Frenk, C. S., et al. 2003, ApJ, 599, 38

Best, P. N., Röttgering, H. J. A., \& Longair, M. S. 2000, MNRAS, 311, 23

Bicknell, G. V., Dopita, M. A., \& O’Dea, C. P. O. 1997, ApJ, 485, 112

Blundell, K. M., \& Rawlings, S. 1999, Nature, 399, 330

Bonnet, H., Abuter, R., Baker, A., et al. 2004, The Messenger, 117, 17

Bullock, J. S., Kolatt, T. S., Sigad, Y., et al. 2001, MNRAS, 321, 559

Capetti, A., Axon, D. J., Macchetto, F. D., Marconi, A., \& Winge, C. 1999, ApJ, 516,187

Croton, D. J., Springel, V., White, S. D. M., et al. 2006, MNRAS, 365, 11

De Breuck, C., van Breugel, W., Röttgering, H. J. A., \& Miley, G. 2000, A\&AS, 143,303

De Breuck, C., van Breugel, W., Röttgering, H., et al. 2001, AJ, 121, 1241

De Breuck, C., Tang, Y., de Bruyn, A. G., Röttgering, H., \& van Breugel, W. 2002, A\&A, 394, 59

De Breuck, C., Neri, R., \& Omont, A. 2003, New Astron. Rev., 47, 285

De Breuck, C., Hunstead, R. W., Sadler, E. M., Rocca-Volmerange, B., \& Klamer, I. 2004, MNRAS, 347, 837

De Young, D. S. 1993, ApJ, 405, L13

Di Matteo, T., Springel, V., \& Hernquist, L. 2005, Nature, 433, 604

Downes, D., \& Solomon, P. M. 1998, ApJ, 507, 615

Holt, J., Tadhunter, C. N., \& Morganti, R. 2003, MNRAS, 342, 227

Holt, J., Tadhunter, C., \& Morganti, R. 2007, New Astron. Rev., 51, 185

Hopkins, P. F., Hernquist, L., Cox, T. J., et al. 2005, ApJ, 630, 705

Humphrey, A., Villar-Martín, M., Fosbury, R., et al. 2007, MNRAS, 1462

Inskip, K. J., Best, P. N., Rawlings, S., et al. 2002a, MNRAS, 337, 1381

Inskip, K. J., Best, P. N., Röttgering, H. J. A., et al. 2002b, MNRAS, 337, 1407

Inskip, K. J., Villar-Martín, M., Tadhunter, C., Holt, J., \& Morganti, R. 2007,

New Astron. Rev., 51, 47

Iwamuro, F., Motohara, K., Maihara, T., et al. 2003, ApJ, 598, 178

Jarvis, M. J., Rawlings, S., Eales, S., et 1. 2001, MNRAS, 326, 1585

Kauffmann, G., Heckman, T. M., Tremonti, C., et al. 2003, MNRAS, 346, 1055

Laing, R. A., \& Bridle, A. H. 2002, MNRAS, 336, 1161

Martini, P. 2004, Invited review to appear in Carnegie Observatories Astrophysics Series, Vol. 1: Coevolution of Black Holes and Galaxies, ed. L. C. Ho (Cambridge: Cambridge Univ. Press)

[arXiv: astro-ph/0304009]

McCarthy, P. J., Baum, S. A., \& Spinrad, H. 1996, ApJS, 106, 281

Morganti, R., Tadhunter, C. N., \& Oosterloo, T. A. 2005, A\&A, 444, L9

Murgia, M., Fanti, C., Fanti, R., et al. 1999, A\&A, 345, 769

Nelson, C. H., \& Whittle, M. 1996, ApJ, 465, 96

Nesvadba, N. P. H., Lehnert, M. D., Eisenhauer, F., et al. 2006, ApJ, 650, 693

Nesvadba, N. P. H., Lehnert, M. D., Genzel, R., et al. 2007, ApJ, 657, 725

Nesvadba, N. P. H., Lehnert, M. D., et al., in preparation

O'Dea, C. P., de Vries, W. H., Koekemoer, A. M., et al. 2002, AJ, 123, 2333

O'Dea, C. P., Gallimore, J., Stanghellini, C., Baum, S. A., \& Jackson, J. M. 2005, AJ, 129, 610

Owsianik, I., \& Conway, J. E. 1998, A\&A, 337, 69

Papadopoulos, P. P., Röttgering, H. J. A., van der Werf, P. P., et al. 2000, ApJ, 528,626

Pedlar, A., Dyson, J. E., \& Unger, S. W. 1985, MNRAS, 214, 463

Pounds, K. A., Reeves, J. N., King, A. R., et al. 2003, MNRAS, 345, 705

Readhead, A. C. S., Taylor, G. B., Pearson, T. J., \& Wilkinson, P. N. 1996, ApJ, 460,634

Seymour, N., et al. 2007, ApJS, accepted [arXiv: astro-ph/0703224]

Silk, J., \& Rees, M. J. 1998, A\&A, 331, L1

Sulentic, J. W., Marziani, P., \& Dultzin-Hacyan, D. 2000, ARA\&A, 38, 521

Tadhunter, C. N. 1991, MNRAS, 251, 46

Tadhunter, C. 2007, New Astron. Rev., 51, 153

Tadhunter, C., Wills, K., Morganti, R., Oosterloo, T., \& Dickson, R. 2001, MNRAS, 327, 227

Tody, D. 1993, in Astronomical Data Analysis Software and Systems II, ed. R. J. Hanisch, R. J. V. Brissenden, \& J. Barnes (San Francisco: ASP), 173 van Breugel, W. 1984, VLBI and Compact Radio Sources, IAU Symp., 110, 59 Villar-Martín, M., Binette, L., \& Fosbury, R. A. E. 1999, A\&A, 346, 7

Villar-Martín, M., Vernet, J., di Serego Alighieri, S., et al. 2002, MNRAS, 336, 436

Villar-Martín, M., Vernet, J., di Serego Alighieri, S., et al. 2003, MNRAS, 346, 273

Villar-Martín, M., Sánchez, S. F., Humphrey, A., et al. 2007, MNRAS, 378, 416 Wellman, G. F., Daly, R. A., \& Wan, L. 1997, ApJ, 480, 96

Willott, C. J., Rawlings, S., Blundell, K. M., Lacy, M., \& Eales, S. A. 2001, MNRAS, 322, 536 